\title{
An Improved Description of the Dielectric Breakdown in Oxides Based on a Generalized Weibull distribution
}

\author{
U. M. S. Costa and V. N. Freire \\ Universidade Federal do Ceará, Departamento de Física, Caixa Postal 6030, \\ Campus do Pici, 60455-900 Fortaleza, Ceará, Brazil \\ L. C. Malacarne, R. S. Mendes and S. Picoli Jr. \\ Universidade Estadual de Maringá, Departamento de Física, \\ 87020-900 Maringá, Paraná, Brazil \\ E. A. de Vasconcelos, E. F. da Silva Jr. \\ Universidade Federal de Pernambuco, Departamento de Física, \\ Cidade Universitária, 50670-901 Recife, Pernambuco, Brazil.
}

(Dated: February 6, 2008)

\begin{abstract}
In this work, we address modal parameter fluctuations in statistical distributions describing charge-to-breakdown $\left(Q_{B D}\right)$ and/or time-to-breakdown $\left(t_{B D}\right)$ during the dielectric breakdown regime of ultra-thin oxides, which are of high interest for the advancement of electronic technology. We reobtain a generalized Weibull distribution ( $q$-Weibull), which properly describes $\left(t_{B D}\right)$ data when oxide thickness fluctuations are present, in order to improve reliability assessment of ultra-thin oxides by time-to-breakdown $\left(t_{B D}\right)$ extrapolation and area scaling. The incorporation of fluctuations allows a physical interpretation of the $q$-Weibull distribution in connection with the Tsallis statistics. In support to our results, we analyze $t_{B D}$ data of $\mathrm{SiO}_{2}$-based $\mathrm{MOS}$ devices obtained experimentally and theoretically through a percolation model, demonstrating an advantageous description of the dielectric breakdown by the $q$-Weibull distribution.
\end{abstract}

PACS numbers: 71.20.Nr,71.30.+h, 77.22.Jp, 85.40.-e,02.50.Cw

\section{INTRODUCTION}

The International Technology Roadmap for Semiconductors indicates the need to decrease the $\mathrm{SiO}_{2}$ gate thickness to less than $3 \mathrm{~nm}$ to pursue the continuity of the Moore's Law [1]. Research efforts to maintain this trend have been focusing on: $(i)$ the advantageous substitution of $\mathrm{SiO}_{2}$ by high dielectric constant materials to allow equivalent gate dielectric oxide thickness bellow 1 $\mathrm{nm}$ 2]; (ii) a better understanding of the fractal regime of ultra-thin gate dielectric oxide growth, where spatial inhomogeneities or fluctuations are important [3, 4] considerations for the control of dielectric breakdown conditions. Consequently, the reliability of ultra-thin oxides for ultra-large scale integration is one of the most important concerns in the domain of electronics miniaturization nowadays [5, 6, 7].

Two quantities which are experimentally measured to assess the reliability of a metal-oxide-semiconductor (MOS) device are: the charge-to-breakdown $Q_{B D}$, defined as the time-integrated current density which flows until breakdown occurs, and the time-to-breakdown $t_{B D}$, defined as the samples' lifetime. These quantities are statistically distributed and are usually assumed to be Weibull distributed [8]. In particular, accurate assessment of reliability of ultra-thin oxides is seriously affected by fluctuations of oxide thickness. These fluctuations cause a deviation from the Weibull distribution, which is easily observed by a curvature in a graph of $\ln [-\ln [1-F(t)]]$ versus $\ln [t]$, being
$F(t)=\int_{0}^{t} P(x) d x$ the cumulative distribution. Extrapolation of the slope of this plot from higher failure percentiles to lower failure percentiles can lead to serious errors in reliability assessment, as shown by $\mathrm{Wu}$ et al. [5].

Deviations from a given distribution can be generated by taking scale mixtures of it 9 ]. For instance, the scale parameter of the exponential and Gaussian distributions can be averaged by a gamma distribution. In these two cases, the deviation induced by the fluctuation in this parameter has been successfully employed in the description of physical situations, such as those related with nonexponential decay [10, 11] and turbulence 12, 13]. Furthermore, this average process has also been connected with the entropic parameter $q$ in the context of Tsallis statistics 10, 11, 12. By taking the fluctuations of the modal parameter of the Weibull distribution into account, we obtain a generalized Weibull distribution which properly describes $t_{B D}$ data when oxide fluctuations are present, thus improving reliability assessment of ultra-thin oxides by $t_{B D}$ extrapolation and area scaling. This generalized Weibull distribution, without focusing fluctuations, was discussed and applied in Ref. [14] to other systems.

In the following section we present the generalized Weibull distribution employed here. The application of this distribution to the description of the dielectric breakdown in oxides is considered in section 3 . The last section is dedicated to our conclusions. 

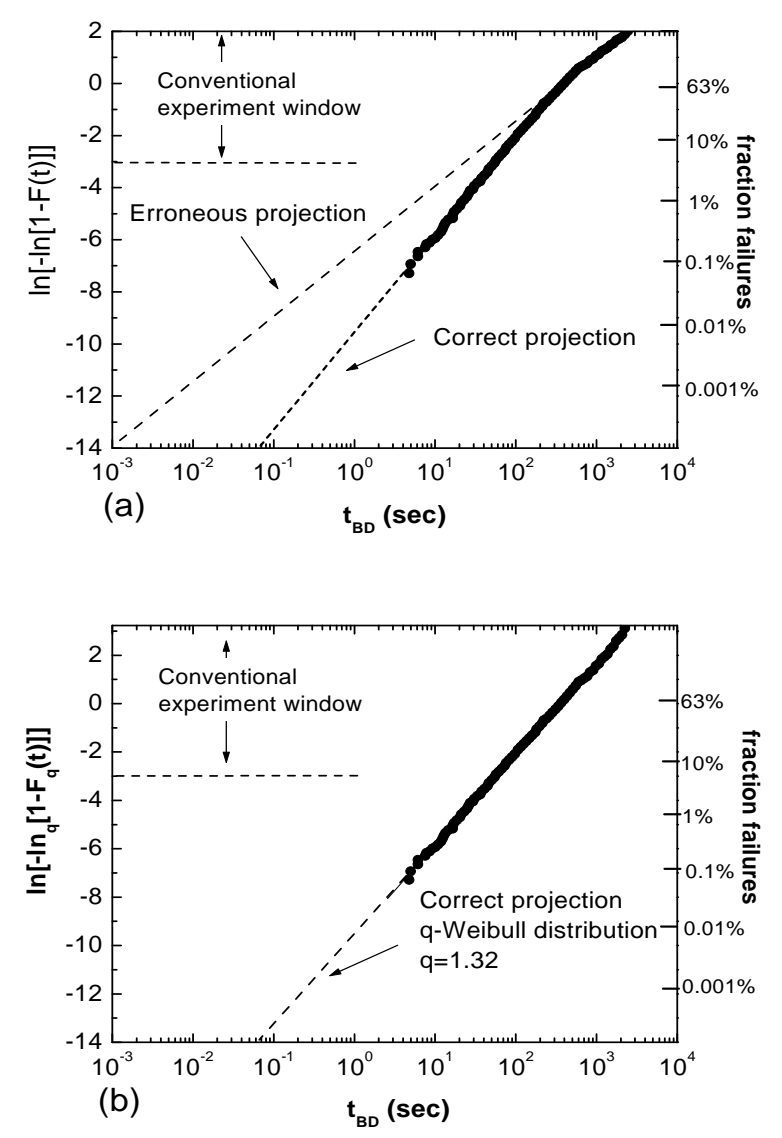

FIG. 1: (a) Graph of $\ln [-\ln [1-F(t)]]$ versus $t_{B D}$ for 4000 ultra-thin oxide MOS capacitors. The data were obtained from the work of $\mathrm{Wu}$ et al. [5]; (b) the same data as in (a) but depicted in a $q$-Weibull plot in which a correct $t_{B D}$ extrapolation is very clear.

\section{II. $q$-WEIBULL DISTRIBUTION}

The Weibull distribution often used in the statistical description in the study of the time-to-breakdown in electronic devices [15] is given by

$$
P_{1}(x)=\frac{\beta}{x_{0}}\left(\frac{x}{x_{0}}\right)^{\beta-1} \exp \left[-\left(\frac{x}{x_{0}}\right)^{\beta}\right],
$$

where the parameter $x_{0}$ is the modal value that corresponds to $63,2 \%$ of the samples' lifetime. The parameter $\beta$ is the Weibull slope and defines the time-to-breakdown spreading degree. In order to incorporate the referred fluctuation, we consider a Weibull compound distribution $p(x ; \theta)=\beta \theta x^{\beta-1} \exp \left(-\theta x^{\beta}\right)$. Here, $\theta=x_{0}^{-\beta}$, with $\beta$ fixed and $x_{0}$ changing so that the new parameter $\theta$ is a stochastic variable ruled by the gamma probability density function

$$
p(\theta)=\frac{\delta^{r} \theta^{r-1} e^{-\delta \theta}}{\Gamma(r)} \quad(\theta, r, \delta>0)
$$
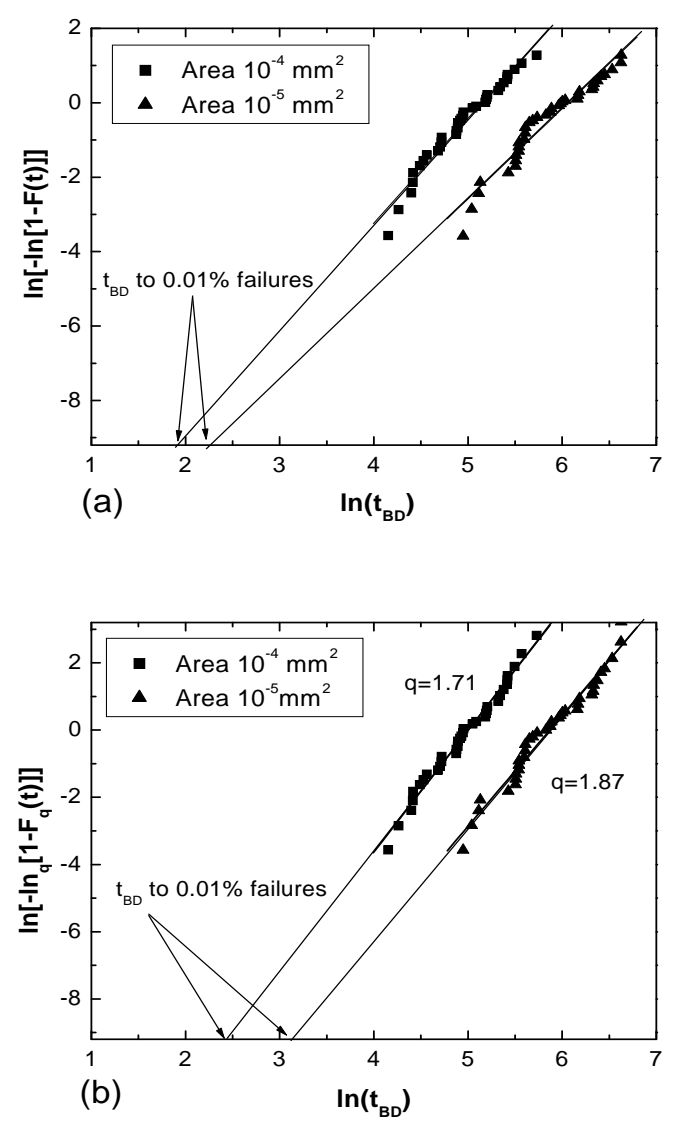

FIG. 2: (a) Graph of $\ln \left[-\ln _{q}\left[1-F^{d}(t)\right]\right]$ versus $\ln (t)$ for a $6.9 \mathrm{~nm}$ sample oxides subjected to a $E_{O x}=12.7 \mathrm{MV} / \mathrm{cm}$ applied field. The samples have areas of $10^{-4}$ and $10^{-5} \mathrm{~mm}^{2}$. The data were obtained from the work of Teramoto et al.) [20]; (b) the same data as in (a) but depicted in a $q$-Weibull plot showing improved area scaling.

Therefore, the average in the stochastic parameter $\theta$,

$$
\langle p(x ; \theta)\rangle_{\theta}=\int_{0}^{\infty} p(x ; \theta) p(\theta) d \theta
$$

leads to 16

$$
\langle p(x ; \theta)\rangle_{\theta}=r \delta^{r} \beta x^{\beta-1}\left(x^{\beta}+\delta\right)^{-(r+1)} .
$$

Note that by naming this average $P_{q^{\prime}}(x)$, it can be written as

$$
P_{q^{\prime}}(x)=\frac{\beta\left(2-q^{\prime}\right)}{\tilde{x}_{0}}\left(\frac{x}{\tilde{x}_{0}}\right)^{\beta-1} \exp _{q^{\prime}}\left[-\left(\frac{x}{\tilde{x}_{0}}\right)^{\beta}\right],
$$

where we used the $q$-exponential function defined as $\exp _{q^{\prime}}(-a x) \equiv\left[1-\left(1-q^{\prime}\right) a x\right]^{1 /\left(1-q^{\prime}\right)}$ if $1-\left(1-q^{\prime}\right) a x \geq 0$, and $\exp _{q^{\prime}}(-a x) \equiv 0$ if $1-\left(1-q^{\prime}\right) a x<0$, with $q^{\prime}=(2+r) /(1+r)$ and $\tilde{x}_{0}^{\beta}=[\delta /(r+1)]$.

By comparing Eq. (1) with Eq. (5) we verify that $P_{q^{\prime}}(x)$ is a generalization of the Weibull distribution in the same 


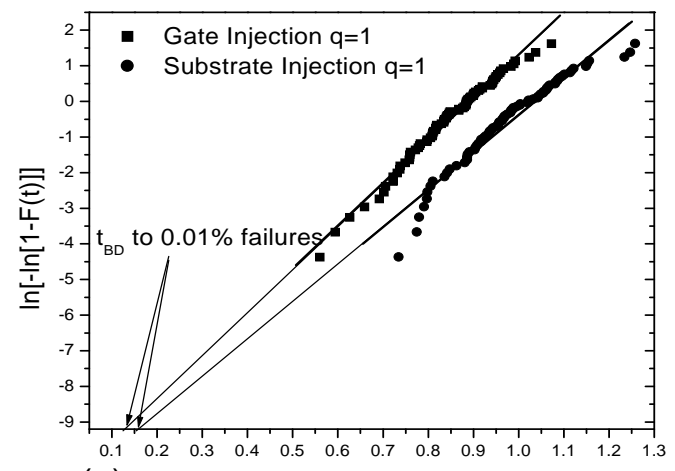

(a)

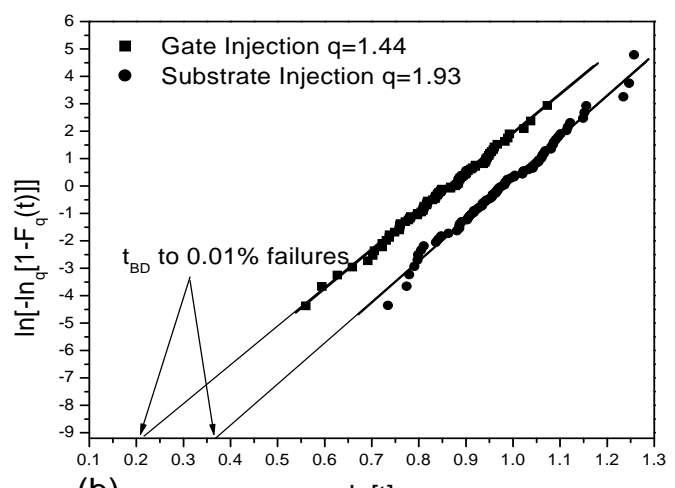

(b)

$\ln [\mathrm{t}]$

FIG. 3: (a) Graph of $\ln \left[-\ln \left[1-F^{d}(t)\right]\right]$ versus $\ln (t)$ from the percolation simulations as performed by Sombra et al. 21] for gate (squares) and substrate (dots) injection adjusted with Weibull distributions; (b) the same data as in (a) but depicted in a $q$-Weibull plot showing improved fitting.

sense that the $q$-exponential function generalizes the exponential one. In view of this observation and following Ref. 14], we refer to $P_{q^{\prime}}(x)$ as $q$-Weibull distribution. We also remark that $P_{q^{\prime}}(x)$ contains a broad class of distributions as particular cases. In fact, in the limit $q^{\prime} \rightarrow 1$ it reduces to the Weibull distribution, for $\beta \rightarrow 1$ it gives the $q$-exponential distribution, and when $q^{\prime} \rightarrow 1$ and $\beta \rightarrow 1$ it becomes the exponential distribution. Furthermore, from Eq.(4) we verify two power law regimes, one for small $x, x^{\beta-1}$, and another for large $x, x^{-(1+r \beta)}$. Notice also that $\int_{0}^{\infty} P_{q^{\prime}}(x) d x$ exists and is equal to one when $q^{\prime}<2$.

Before addressing our discussion to the application of the $q$-Weibull distribution concerning experimental and simulation results, we give a connection of this distribution with the Tsallis statistics. The first step towards verifying this connection is based on the fact that the $q$-exponential can be viewed as a signature of the Tsallis statistics, since it basically replaces the exponential in the canonical distribution. In fact, this canonical distribution is obtained when the Tsallis entropy
$S_{q} \equiv\left(1-\sum_{i=1}^{W} p_{i}^{q}\right) /(1-q)$ is maximized subjected to appropriate constraints [17]. Here, $p_{i}$ is the probability of the $i$ th state, $W$ is the number of accessible states, and $q$ is a real parameter that rules the degree of generalization of the theory (when $q \rightarrow 1$ we recover the usual entropy). On the other hand, the q-exponential function can be obtained from the average of the exponential function by using the gamma distribution, Eq. (22) 18]. Thus, the parameter $q$ occurring in the Tsallis statistics is shown to be entirely induced by the fluctuations of the parameter of the usual exponential distribution [10]. Following this interpretation, the average process employed to obtain $P_{q^{\prime}}(x)$ characterizes an implicit connection with the Tsallis statistics.

\section{APPLICATION TO DIELECTRIC BREAKDOWN}

To apply our distribution, Eq. (5), to investigate experimental and numerical simulated data, we consider the cumulative distribution for $P_{q^{\prime}}(x)$, i.e.,

$$
F_{q}(t)=1-\exp _{q}\left[-\left(\frac{t}{\alpha}\right)^{\beta}\right],
$$

with $q=1 /\left(2-q^{\prime}\right)$ and $\alpha=\tilde{x}_{0} /\left(2-q^{\prime}\right)^{1 / \beta}$. In connection with the $q$-exponential function, the $q$-logarithm function is usually defined as 17.

$$
\ln _{q}(x) \equiv \frac{x^{1-q}-1}{1-q},
$$

thus $\ln _{q}\left[\exp _{q}(x)\right]=\exp _{q}\left[\ln _{q}(x)\right]=x$. By using Eq. (6), we obtain that the graph of $\ln \left[-\ln _{q}\left(1-F_{q}(t)\right)\right]$ versus $\ln (t)$ gives a straight line since

$$
\ln \left[-\ln _{q}\left[1-F_{q}(t)\right]\right]=\beta \ln (t)-\beta \ln (\alpha) .
$$

Therefore, if a cumulative distribution relative to the dielectric breakdown for a given set of data, $F^{d}(t)$, is well described by the $q$-Weibull distribution, then the $q$ parameter present in the $q$-logarithm is obtained through a linear adjustment, and the parameter $\beta$ is the slope of the graph. This procedure introduced here will be referred as a $q$-Weibull plot.

To compare the usefulness of the Weibull and the $q$ Weibull distributions, we analyze experimental [19] and simulation results. Figure 1(a), which is adapted from the work of $\mathrm{Wu}$ et al. [5], shows a curvature in a graph $\ln [-\ln [1-F(t)]]$ versus $\ln [t]$. The variation of this slope is associated with oxide thickness fluctuations. In fact, $\mathrm{Wu}$ et al. [5] were able to generate this slope variation by performing simulations in which the oxide thickness obeyed a Gaussian distribution. Extrapolation of the slope of this plot from higher failure percentiles (conventional experimental window) to lower failure percentiles leads to an error in $t_{B D}$ projection and, consequently, in projection of reliability. It is necessary to use large 
and time-consuming sample sizes (thousands of devices) in order to obtain $t_{B D}$ distributions down to lower percentiles and avoid erroneous projections. The same $t_{B D}$ data can be correctly fitted in the entire range of percentiles by the $q$-Weibull distribution, as shown in Fig. 11(b). In the $q$-Weibull plot, the data follow a straight line. We conclude that, by taking the fluctuations of the modal parameter of the Weibull distribution into account, one obtains a statistical distribution which completely describes $t_{B D}$ data when oxide fluctuations are present. Therefore, the $q$-Weibull distribution allows improved $t_{B D}$ projection for ultra-thin oxides and does not require a very large number of samples, i.e., correct $t_{B D}$ extrapolation can be performed in the conventional experimental window. This is a striking benefit brought by the $q$-Weibull distribution.

In addition to correct $t_{B D}$ projection, we have found that the $q$-Weibull also improves area scaling, another important consideration for reliability studies [15]. In Fig. 2(a), we have used the data from the work of Teramoto et al. [20]. They studied the time-dependent dielectric breakdown for $6.9 \mathrm{~nm}$ thin $\mathrm{SiO}_{2}$ oxides with samples areas of $10^{-4}$ and $10^{-5} \mathrm{~mm}^{2}$, and subjected to a $E_{O x}=12.7 \mathrm{MV} / \mathrm{cm}$ applied field. It is clear, when comparing Fig. 2(a) and Fig. 2(b), that the $q$-Weibull distribution gives a better area scaling than the Weibull one, another remarkable benefit.

When $t_{B D}$ data are plotted in a limited range of percentiles, the differences between the $q$-Weibull and the Weibull distributions become less apparent, but can still be seen. As an example, we consider the simulations performed by Sombra et al. 21]. They developed a percolation model to describe the dielectric breakdown of a MOS capacitor investigating effects of bias polarity, ox- ide film thickness and electric field strength. The hot electron injection is either through the gate or the substrate. In Fig. 31 a) we have performed a linear fit of the simulation data of Sombra 21] with the Weibull distribution, while in Fig. 3(b) we depict the same data but with a linear fit through the $q$-Weibull distribution. One can see once more that the $q$-Weibull distribution gives a better fit than the Weibull one, for small and large $t_{B D}$.

\section{CONCLUSIONS}

In summary, we conclude that: ( $i$ ) fluctuation on the modal value is a mechanism to explain deviations from the Weibull distribution in reliability studies of electronics devices; ( $i i)$ this fluctuation leads to a generalization of the Weibull distribution ( $q$-Weibull distribution) and a connection with the Tsallis statistics; and (iii) the generalized Weibull distribution leads to a better adjustment of experimental and simulated data in comparison with the Weibull one, giving improved $t_{B D}$ extrapolation and area scaling. Finally, the results presented here can be very useful for an improved description of the dielectric breakdown in the high dielectric constant materials [2] which will be present in future generations of advanced MOS devices.

The authors would like to acknowledge the financial support received from the Brazilian National Research Council (CNPq) under contract CNPQ-NanoSemiMAt \#550.015/01-9, and the Ministry of Planning (FINEP) through CTPETRO under contracts \# 65.00.02.80.00 and \#5000013/01-2, during the development of this work.
[1] The International Technology Roadmap for Semiconductors (Semiconductor Industry Association, San Jose, CA, 2002), see also http://public.itrs.net for the most recent updates.

[2] G. D. Wilk, R. M. Wallace, and J. M. Anthony, J. Appl. Phys. 89, 5243 (2001); R. A. MacKee, F. J. Walker, M. F. Chisholm, Phys. Rev. Lett. 81, 3014 (1998); R. A. MacKee, F. J. Walker, M. F. Chisholm, Science 293, 468 (2001); V. Fiorentini and G. Gulleri, Phys. Rev. Lett. 89, 6101 (2002); D. Muller, T. Sorsch, S. Moccio, F. Baumann, K. Evans-Lutterdodt, and G. Timp, Nature 399, 758 (1999).

[3] L. Niemeyer, L. Pietronero, and H. J. Wiesmann, Phys. Rev. Lett. 52, 1033 (1984); M. A. F. Gomes, E. F. da Silva Jr., and J. A. Aguiar, Semicond. Sci. Techn., 10, 1037 (1995).

[4] H. J. Wiesmann, and H. R. Zeller, J. Appl. Phys. 60, 1770 (1986).

[5] E. Y. Wu, J. H. Stathis and L-K. Han, Semicond. Sci. Technol. 15, 425 (2000).

[6] R. Degreave, B. Kaczer and G. Groeseneken, Microelectron. Reliab. 39, 1445 (1999).
[7] M. L. Green, E. P. Gusev, R. Degreave and E. L. Garfunkel, J. Appl. Phys. 90, 2057 (2001).

[8] D. R. Wolters, and J. F. Verwey, in Instabilities in Silicon Devices, edited by G. Barbottin and A. Vapaile (Elsevier, North Holland,1986), p. 315.

[9] B. V. Gnedenko and V. Yu. Korolev, Random Summation: Limit Theorems and Applications (CRC, Boca Raton, 1996).

[10] G. Wilk and Z. Wlodarczyk, Phys. Rev. Lett 84, 2770 (2000).

[11] G. Wilk and Z. Wlodarczyk, Physica A 290, 55 (2001).

[12] C. Beck, Phys. Rev. Lett. 87, 180601 (2001).

[13] G. M. Batanov et al, Plasma Phys. Rep. 28, 111 (2002).

[14] S. Picoli Jr., R. S. Mendes, and L. C. Malacarne, Physica A 324, 678 (2003).

[15] R. Degraeve, B. Kaczer, and G. Groeseneken, Semicond. Sci. Technol. 15, 436 (2000).

[16] N. L. Johnson and S. Kotz, Distribution in Statistics: Continous Univariante Distribution-1, New York, John Wiley and Sons, 1970.

[17] C. Tsallis, J. Stat. Phys. 52, 479 (1988); C. Tsallis, R. S. Mendes and A. R. Plastino, Physica A 261, 534 
(1998); C. Tsallis, in: S. Abe, Y. Okamoto (Eds.), Nonextensive Statistical Mechanics and Its Application, Lecture Notes in Physics, Springer, Heidelberg, 2001; See http://tsallis.cat.cbpf.br for periodically updated bibliography on the subject.

[18] Strictly speaking, this average process only leads to $q>$ 1 ,which is just the relevant case to this work.

[19] The experimental data were obtained through direct scanning of the figures and then recovering the data using the Opti-Graph program.

[20] A. Teramoto,H. Umeda, K. Azamawari, K. Kobayashi, K. Shiga, J. Komori, Y. Ohno, and A. Shigetomi, Microelectron. Reliab., 41, 47 (2001).

[21] S. S. Sombra, U. M. S. Costa, V. N. Freire, E. A. de Vasconcelos, E. F. da Silva Jr., Physica A 305, 351 (2002). 